\section{Edible tools to fight Alzheimer's}

Two separate groups of researchers are now pursuing edible options for the treatment or prevention of Alzheimer's disease. One study carried out by HyunSoon Kim (Korea Research Institute of Bioscience and Biotechnology) and colleagues showed that tomatoes may serve as effective carriers for an oral vaccine against $\beta$-amyloid in mice. Accumulation of $\beta$-amyloid protein in the brain leads to neuronal death, which is thought to underlie the neurodegeneration characteristic of Alzheimer's disease. The other group, led by Richard Wurtman at Massachusetts Institute of Technology (Cambridge, MA), found that dietary supplements of docosahexaenoic acid (DHA), uridine and choline improved learning and memory in gerbils.

Kim's group used bacteria-mediated transformation to generate tomato plants that expressed human $\beta$-amyloid protein. They then made a soluble extract and administered it orally to mice once a week for 3 weeks, with a booster 7 weeks after the initial administration. The mice had a strong immune response to the booster and produced antibodies to the foreign human $\beta$-amyloid protein (Biotechnol. Lett. doi:10.1007/s10529-008-9759-5; published online 5 July 2008).

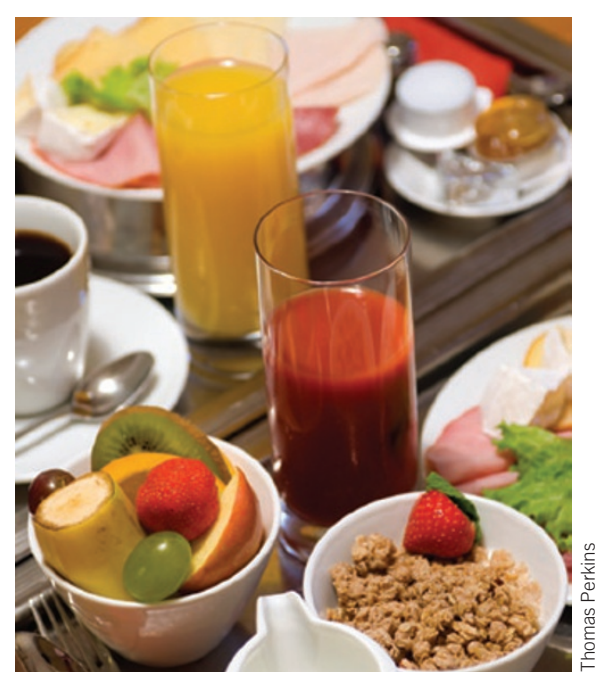

Tomatoes were chosen for several reasons. $\beta$-amyloid is toxic to animal cells and is difficult to produce using E. coli or yeast cells, which are more commonly used in such applications. In addition, tomatoes can be consumed raw, which is an advantage because heat treatment may compromise the immunogenicity of the $\beta$-amyloid antigen. One disadvantage of the tomato-based system is that tomatoes contain relatively small amounts of native protein $(0.7 \%)$ and can express even less foreign protein. Kim's group is currently investigating the possibility of increasing the expression level of foreign proteins in the tomatoes in order to increase the vaccine's strength. It is not yet known how or whether the vaccine will affect existing plaques of $\beta$-amyloid in brain tissue.

Wurtman's group used a different approach, targeting restoration of the synapses, which deteriorate in Alzheimer's disease. They had previously shown that when administered to gerbils via dietary supplements, the combination of DHA, choline and uridine resulted in increases in brain phosphatides and certain synaptic proteins. All three compounds are normally found in the bloodstream and are precursors to the fatty molecules that compose cell membranes. The new study found that performance in tests of cognitive function improved in gerbils given the dietary supplements (FASEB J. doi:10.1096/fj.08112425; published online 7 July 2008), linking increases in synaptic membrane content with improvements in learning and memory.

The two ideas together put a whole new spin on the glass of tomato juice and vitamin pill you might have had with your breakfast! Monica Harrington

\title{
NEW CLUES INTO THE SOURCE OF STDS
}

Little is known about what triggers sudden infant death syndrome (SIDS), the leading cause of death in the US in babies aged 1-12 months. Though various environmental risk factors have been identified, including sleep position, insufficient prenatal care and maternal substance abuse, the molecular mechanisms of the syndrome are poorly understood. Recent postmortem studies in SIDS victims have indicated that serotonin dysfunction may be involved, but attempts to develop disease models relying on serotonin deficits have so far been unsuccessful.

A new study led by Cornelius Gross of the European Molecular Biology Laboratory (Monterotondo, Italy) may begin to shed light on the relationship between SIDS and serotonin. The team used mice that were genetically modified to overexpress the serotonin receptor $1 \mathrm{~A}$ in the brainstem (Science 321, 130-133; 2008). In mammals, this receptor regulates serotonin expression through a negative feedback mechanism. Increasing expression of this receptor reduces serotonin neuron firing and decreases heart rate, body temperature and respiration.

Like human SIDS victims, mutant mice seemed healthy at first, and their baseline heart rates and body temperatures were normal. When researchers used telemetric devices to monitor mice continuously, however, they found that mice sporadically suffered autonomic 'crises' in which their heart rates and body temperatures dropped abruptly. Data from infants who happened to have been monitored during SIDS events indicate that similar symptoms occur in humans, as well. Most mice died from such crises, and death occurred early in life, well before mice were 3 months old. The similarities between modified mice and human SIDS victims suggest that the mice would be an appropriate model for the condition.

The sudden death in mice came as a surprise to researchers, as this effect does not occur in mice lacking serotonin neurons entirely. This would suggest that an imbalanced serotonin system is more lethal than a nonexistent one. Additionally, the team's findings show that sudden death can be caused by an animal's predisposition alone and is not necessarily triggered by environmental stressors that parents, for example, might be able to control.

It is not clear what caused mice to enter crises, but the scientists speculate that the effects may be related to rapid changes in serotonin neuron activity, such as those that occur during the transition from sleep to wakefulness. The group intends to continue exploring the connection between sleep and sudden death. Karen Marron 